\title{
Interface-engineered templates for molecular spin memory devices
}

Karthik V. Raman, Alexander M. Kamerbeek, Arup Mukherjee, Nicolae Atodiresei, Tamal K. Sen, Predrag Lazić, Vasile Caciuc, Reent Michel, Dietmar Stalke, Swadhin K. Mandal, Stefan Blügel, Markus Münzenberg \& Jagadeesh S. Moodera

Received 11 May 2012, Accepted 23 October 2012, Published online 23 January 2013

DOI: $10.1038 /$ nature11719

URL: http://www.nature.com/nature/journal/v493/n7433/full/nature11719.html

The use of molecular spin state as a quantum of information for storage, sensing and computing has generated considerable interest in the context of next-generation data storage and communication devices ${ }^{1,2}$, opening avenues for developing multifunctional molecular spintronics ${ }^{3}$. Such ideas have been researched extensively, using singlemolecule magnets ${ }^{4,5}$ and molecules with a metal ion ${ }^{6}$ or nitrogen vacancy ${ }^{7}$ as localized spin-carrying centres for storage and for realizing logic operations ${ }^{8}$. However, the electronic coupling between the spin centres of these molecules is rather weak, which makes construction of quantum memory registers a challenging task ${ }^{9}$. In this regard, delocalized carbon-based radical species with unpaired spin, such as phenaleny ${ }^{10}$, have shown promise. These phenalenyl moieties, which can be regarded as graphene fragments, are formed by the fusion of three benzene rings and belong to the class of open-shell systems. The spin structure of these molecules responds to external stimuli ${ }^{11,12}$ (such as light, and electric and magnetic fields), which provides novel schemes for performing spin memory and logic operations. Here we construct a molecular device using such molecules as templates to engineer interfacial spin transfer resulting from hybridization and magnetic exchange interaction with the surface of a ferromagnet; the device shows an unexpected interfacial magnetoresistance of more than 20 per cent near room temperature. Moreover, we successfully demonstrate the formation of a nanoscale magnetic molecule with a well-defined magnetic hysteresis on ferromagnetic surfaces. Owing to strong magnetic coupling with the ferromagnet, such independent switching of an adsorbed magnetic molecule has been unsuccessful with single-molecule magnets ${ }^{13}$. Our findings suggest the use of chemically amenable phenalenyl-based molecules as a viable and scalable platform for building molecular-scale quantum spin memory and processors for technological development.

The diversity and flexibility of molecular synthesis has given researchers ample freedom to design functional molecules for spintronics. These include molecular magnets ${ }^{14}$, spinfilter molecules ${ }^{15}$, spin-crossover molecules ${ }^{16}$, molecular batteries ${ }^{17}$, molecular conductors $^{10}$, molecular switches ${ }^{12}$, and spacer layers for organic spin valves ${ }^{18}$ and magnetic tunnel junctions ${ }^{19,20}$. Using such synthetic techniques, we have designed a neutral planar phenalenyl-based molecule, zinc methyl phenalenyl (ZMP, $\mathrm{C}_{14} \mathrm{H}_{10} \mathrm{O}_{2} \mathrm{Zn}$; see Fig. $1 \mathrm{a}$ and Methods), that has no net spin. When these molecules are grown on a ferromagnetic surface, interface spin transfer causes a hybridized organometallic supramolecular magnetic layer to develop, which shows a large magnetic anisotropy and spin-filter properties ${ }^{21}$. This interface layer creates a spin-dependent resistance and gives rise to an interface magnetoresistance (IMR) effect. 
Figure 1: Interface magnetoresistance effect.

a

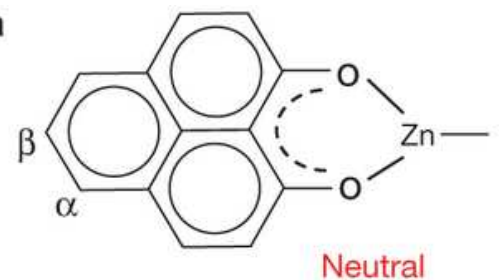

$-\mathrm{e}^{-}||+\mathrm{e}^{-}$

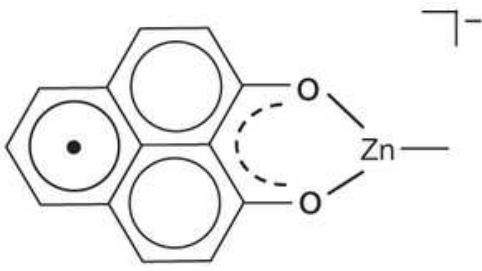

Anionic radical

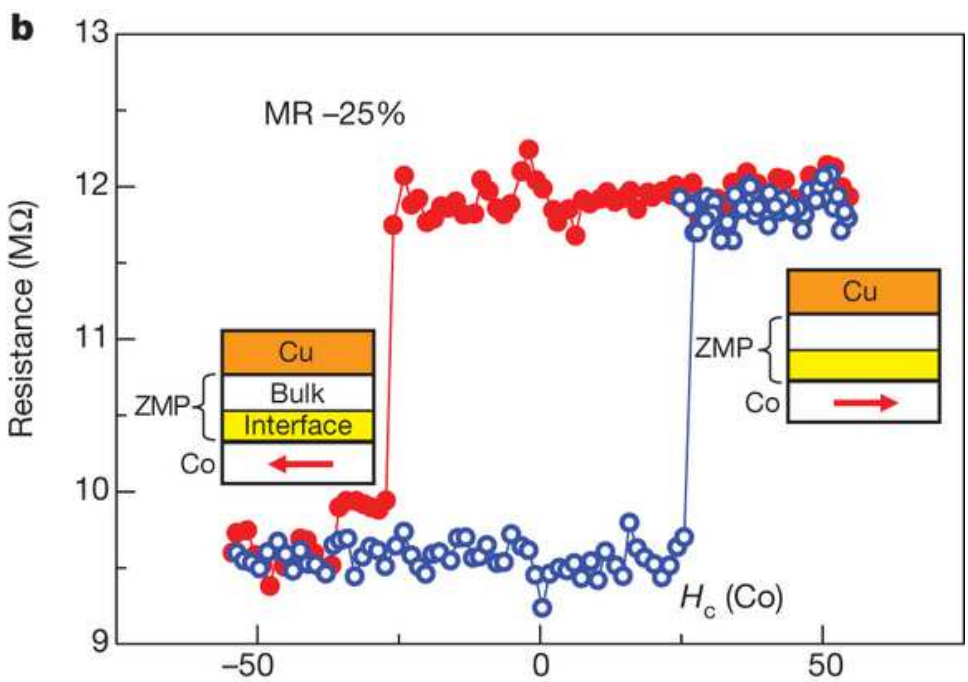

Magnetic field (Oe)

a, Molecular structure of zinc methyl phenalenyl (ZMP) in a neutral state with no net spin (top). Charge transfer processes through hybridization on the ferromagnet surface can change the chemical state of the phenalenyl moiety from neutral to an anionic radical (bottom) with net moment. $\mathbf{b}$, Magnetoresistance measurements (data points) of a device with a single ferromagnetic electrode $(\mathrm{Co}(8 \mathrm{~nm}) / \mathrm{ZMP}(40 \mathrm{~nm}) / \mathrm{Cu}(12 \mathrm{~nm}))$, measured at $15 \mathrm{mV}$ after cooling the device to $4.2 \mathrm{~K}$ in a magnetic field (about -550 Oe). Blue and red data points refer to positive and negative field sweeps respectively. The solid lines are guides to the eye. The magnetoresistance loop corresponds to the switching of the Co magnetization, as shown in the two insets, with respect to the hard magnetic layer forming at the interface (yellow). Left and right insets show the Co magnetization pointing in the negative and positive direction, respectively.

We demonstrate IMR response in a vertical-junction molecular device consisting of a ZMP thin film grown on a (111) textured f.c.c. cobalt (Co) film surface (see Methods). Figure $1 \mathrm{~b}$ shows a magnetotransport measurement on such a device with copper ( $\mathrm{Cu}$ ) as the top contact electrode over a 40-nm ZMP spacer layer. The device is cooled down to $4.2 \mathrm{~K}$ in a magnetic field of -550 Oe. The nonlinear current-voltage $(I-V)$ characteristics of the device (see Supplementary Information) suggest tunnelling as the dominant transport mechanism. A large magnetoresistance signal, close to $25 \%$, is observed when the Co magnetization switches at its coercive field. Here we only show the minor loop corresponding to the switching of one ferromagnetic electrode, while the second electrode is non-magnetic $\mathrm{Cu}$. Furthermore, when the bottom $\mathrm{Co}$ electrode is replaced by $\mathrm{Cu}$, there is no magnetoresistance signal. These observations imply that the magnetoresistance effect arises due to the switching of the Co electrode with respect to a hard magnetic layer, either at the interface or within the bulk of the ZMP film. Bulk magnetism in ZMP can be ruled out (see Supplementary Information), clearly pointing to an interfacial phenomenon as being responsible for the IMR effect.

The IMR response is confirmed by performing magnetoresistance measurements on a standard magnetic junction structure, replacing Cu with Permalloy (Py): Co/ZMP (35 nm)/Py (Fig. 2a). Instead of seeing a 'standard' magnetoresistance signal, a large IMR of $42 \%$ with 
sharp switching at the coercivity $\left(H_{c}\right)$ of Co $( \pm 38 \mathrm{Oe})$ is observed. A low-field scan showed a second independent IMR loop corresponding to the $H_{c}$ of the Py electrode $( \pm 3 \mathrm{Oe})$. The $H_{\mathrm{c}}$ of the ferromagnetic electrodes is confirmed from the anisotropic magnetoresistance (AMR) measurements of the electrodes. These results suggest that the magnetoresistance does not arise from conventional spin-conserved tunnelling/transport through the organic barrier between the two ferromagnetic electrodes ${ }^{20}$. This is further confirmed by inserting an ultrathin layer of $\mathrm{Al}_{2} \mathrm{O}_{3}, 0.7 \mathrm{~nm}$ thick, at the bottom interface to create an interfacemodified junction- $\mathrm{Co} / \mathrm{Al}_{2} \mathrm{O}_{3} / \mathrm{ZMP} / \mathrm{Py}$ - that showed a resistance change only when the $\mathrm{Py}$ magnetization was switched (Fig. 2b). Clearly, the ZMP has to be in direct contact with the ferromagnetic surface to observe IMR. The switching response of the interface layer was characterized in larger magnetic fields (Fig. 3a and b, and Supplementary Information). For $\mathrm{Co} / \mathrm{ZMP} / \mathrm{Py}$ at $4.2 \mathrm{~K}$, the bottom Co/ZMP interface layer switches at about \pm 600 Oe to the low-resistance state (the switching field varied from device to device). After warming up to near room temperature $(\sim 250 \mathrm{~K})$, the device showed a large IMR of $22 \%$ with a lower switching field ( $100 \mathrm{Oe})$ of the interface layer.

Figure 2: Confirmation of IMR.
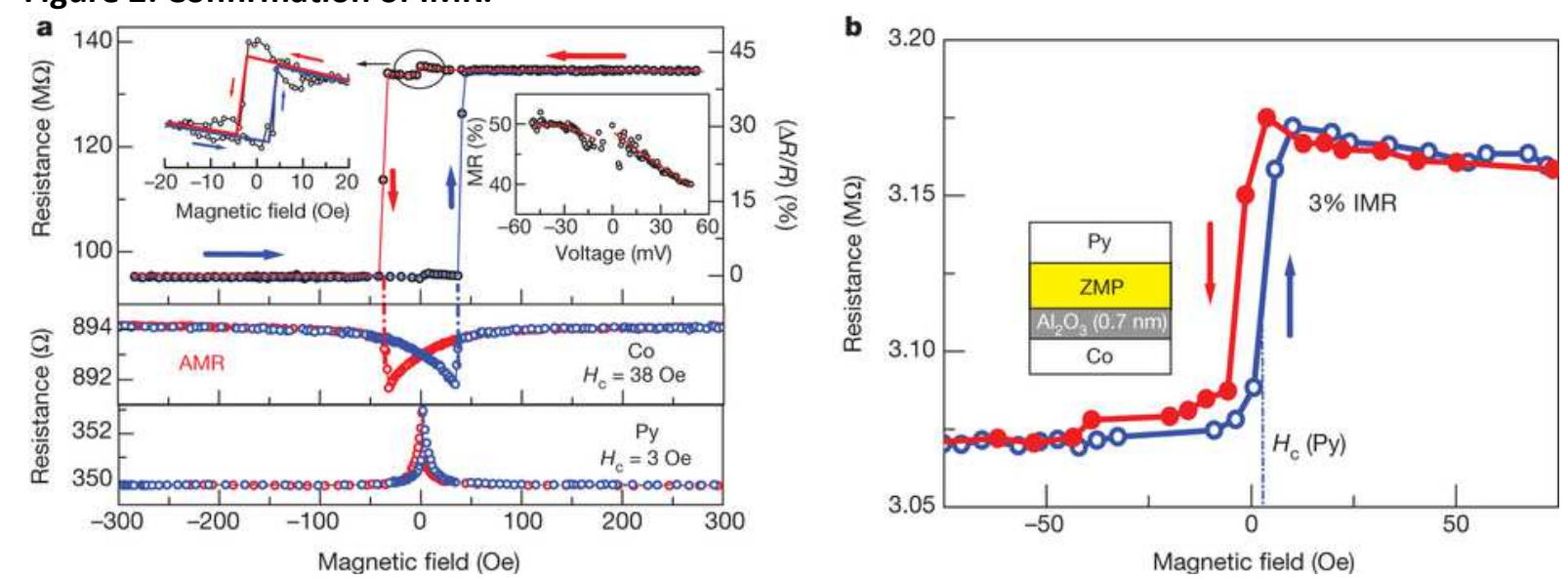

a, Top panel, magnetoresistance measurements (black data points) on a Co $(8 \mathrm{~nm}) / \mathrm{ZMP}(35 \mathrm{~nm}) / \mathrm{Py}$ $(12 \mathrm{~nm}$ ) device (at $25 \mathrm{mV}$ ) show independent IMR response of both the bottom Co/ZMP interface and the top ZMP/Py interface around zero field. Left inset, zoomed-in view showing low field scan. Right inset, IMR of bottom Co/ZMP interface versus applied bias shows a flat response under low bias conditions with a maximum of $50 \%$ in negative bias. Middle and bottom panels show AMR measurements of Co and Py electrode, respectively. b, Magnetoresistance measurements on an interface-modified junction, $\mathrm{Co}(8 \mathrm{~nm}) / \mathrm{Al}_{2} \mathrm{O}_{3}(0.7 \mathrm{~nm}) / \mathrm{ZMP}(30 \mathrm{~nm}) / \mathrm{Py}(12 \mathrm{~nm})$, show 3\% IMR (at 50 $\mathrm{mV}$ ) due only to Py switching (data points). The blue and red curves refer to positive and negative field sweeps, respectively, and arrows show sweep direction.

A similar two-step switching magnetoresistance response has been reported in devices using one ferromagnetic electrode: (Ga,Mn)As/Al ${ }_{2} \mathrm{O}_{3} / \mathrm{Al}$ tunnel junctions ${ }^{22}$. The magnetoresistance in such tunnel devices is referred to as tunnelling anisotropy magnetoresistance (TAMR), which arises from the magnetization-dependent total density of states of the tunnelling electrons at the surface. The observation of TAMR has also been reported in organic spin-valve structures using epitaxial lanthanum strontium manganese oxide electrodes ${ }^{23}$. However, our above experimental observations suggest the origin of IMR in our device to be interfacial and different to that of TAMR. Though the details of the interface phenomenon need further study, we suggest an interface model strengthened by 
$a b$ initio calculations that reasonably explain our experimental observations. Figure $3 c$ and $d$ shows the model: the interface magnetic layer comprises the hybridized Co surface layer with an adsorbed ZMP dimer. The adsorbed dimer forms a pair of magnetic and spin-filter molecules. The Co electrode with spin polarized $s-p$ electrons serves as the spin-polarizer, and the interface magnetic layer with spin-dependent interface resistance serves as the spin analyser, giving rise to IMR.

Figure 3: Interface switching response and interface model.

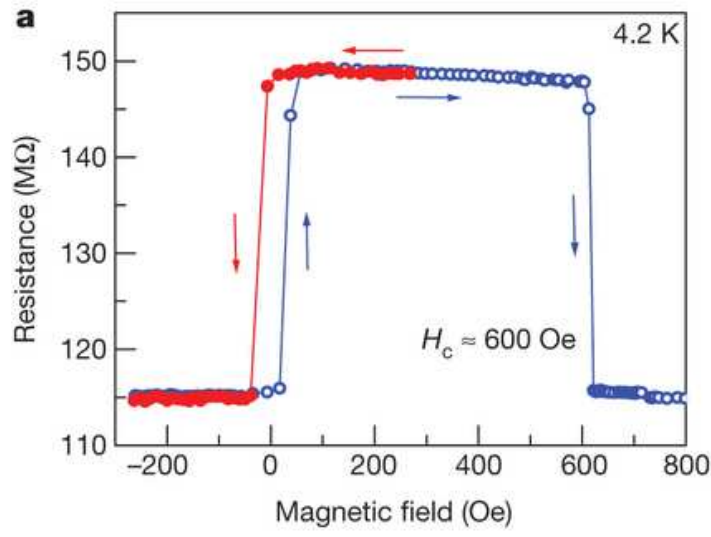

c

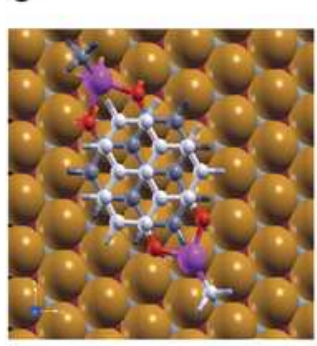

Top view
Spin-filter molecule

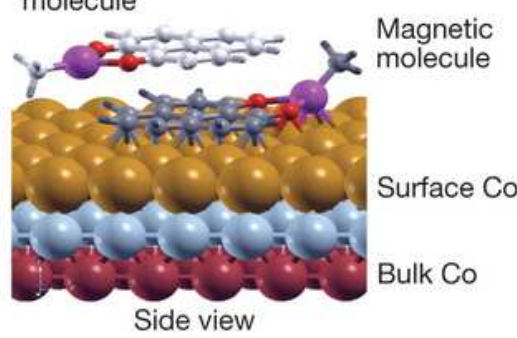

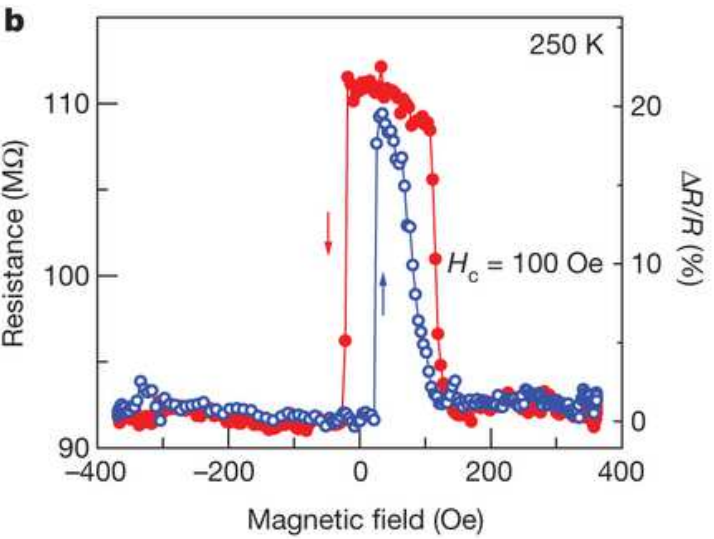

d

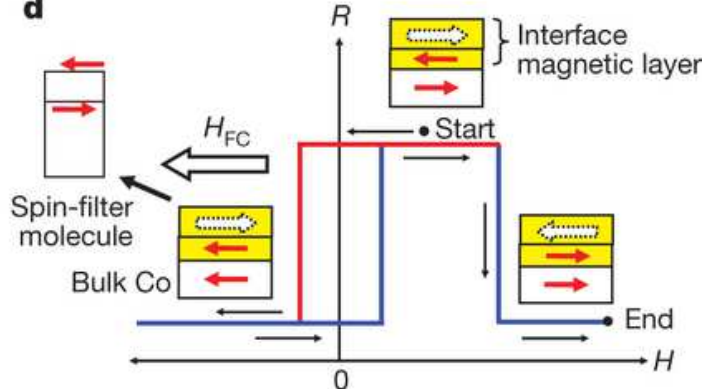

a, $\mathbf{b}$, IMR measurements under high magnetic field on the device Co $(8 \mathrm{~nm}) / \mathrm{ZMP}(35 \mathrm{~nm}) / \mathrm{Py}(12$ $\mathrm{nm})$ : a, at $4.2 \mathrm{~K}$ showing large switching field $\left(H_{\mathrm{c}} \approx 600 \mathrm{Oe}\right)$ of the interface layer; $\mathbf{b}$, at $250 \mathrm{~K}$, showing large IMR of $22 \%$ with reduction in switching field ( $100 \mathrm{Oe}) . \mathbf{c}$, Ab initio model: top view (left) and side view (right) of the relaxed molecules' configuration on a Co(111) surface; grey, carbon; red, oxygen; purple, zinc. The first-layer ZMP molecule ('magnetic molecule') absorbs flat, with six $\alpha$-carbons and the central carbon of the phenalenyl sitting directly on top of Co atoms, while the $\beta$-carbons of the phenalenyl sit in a bridge position between Co atoms. The molecular plane is $\sim 2.1-2.2 \AA$ above the $\mathrm{Co}(111)$ surface (the $\mathrm{C}$ atoms are $2.1 \AA$ above, the $\mathrm{O}$ and $\mathrm{Zn}$ atoms are $2.2 \AA$ above). The second molecule ('spin filter molecule') sits in a staggered configuration over the first adsorbed molecule with a spacing of $\sim 3.26 \AA$, forming a molecular $\pi$-dimer. $d$, Device model: an interface magnetic layer comprises the surface hybridized Co atoms with the molecular dimer on top, forming a magnetic molecule and a tunnel spin-filter molecule. Shown are relative magnetization directions of the interface magnetic layer and the bottom bulk $C o$ at various field values ( $R$, resistance; $H$, magnetic field; arrow labelled $H_{\mathrm{FC}}$ shows direction of field cooling). Arrows in the spin-filter molecule represent the two electron-spin energy levels.

Chemical modification of the ferromagnet/ZMP molecule interface arises owing to charge transfer and hybridization of the molecular orbitals with the $d$-orbitals of the ferromagnet, creating new hybrid metal-organic interface states that directly affect the electronic and magnetic properties of the adsorbed molecule ${ }^{24}$. These interactions strongly depend on the specific molecular structure and morphology ${ }^{25,26}$, influencing the efficiency of spin 
polarization and spin injection at the interface. In the case of ZMP, the planar geometry of the molecule provides an ideal situation for strong orbital overlap leading to $d-p$ hybridization effects. Spin-polarized first-principles calculations using density-functional theory (DFT) were carried out in zero magnetic field on such a hybrid metal-organic interface, comprising five Co(111) layers and either one or two molecular layers of ZMP on top (see Methods and Supplementary Information).

The $p_{z}$ atomic-type orbitals of the planar ZMP molecule hybridize strongly with the $d$ states (mainly with $d_{z^{2}}, d_{x z}$ and $d_{y z}$ orbitals) of the Co atoms, forming hybrid molecule-metal $p_{z}-d$ interface states with bonding and anti-bonding characters ${ }^{24}$. The calculated spinresolved projected density-of-states (PDOS) is shown in Fig. $4 a$ and b. The molecular energy levels of the first adsorbed ZMP molecule are broadened, becoming a component of the metallic hybrid molecule-surface states. In addition, the molecular layer acquires a net moment of $0.11 \mu_{\mathrm{B}}$ oriented anti-parallel to the moment of the hybridized surface layer of Co atoms giving an anti-ferromagnetic $(\mathrm{AFM})$ coupling $\left(J_{\mathrm{Co}-\mathrm{ZMP}}<0\right)$. The moment of the surface Co atoms is lowered to $\sim 1.7 \mu_{\mathrm{B}}$ per atom (bulk moment $\sim 1.9 \mu_{\mathrm{B}}$ per atom).

Figure 3: Interface switching response and interface model.

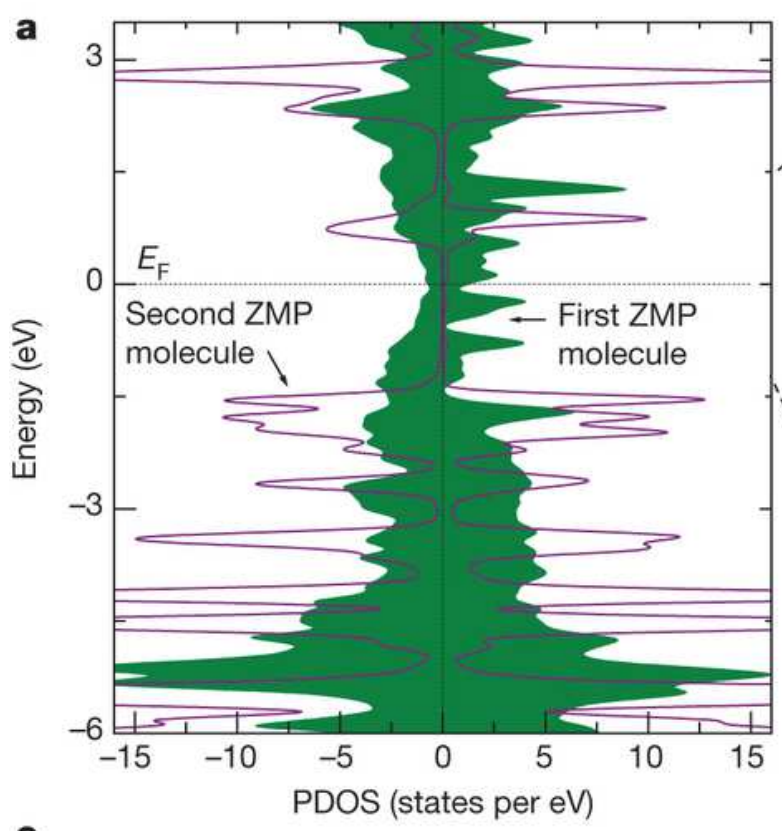

C

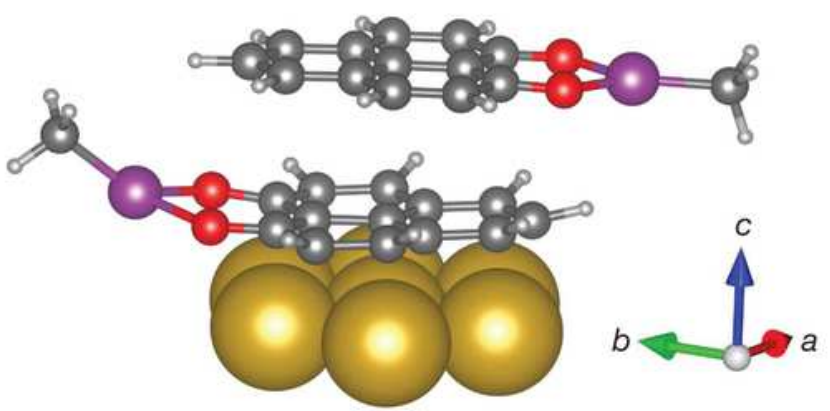

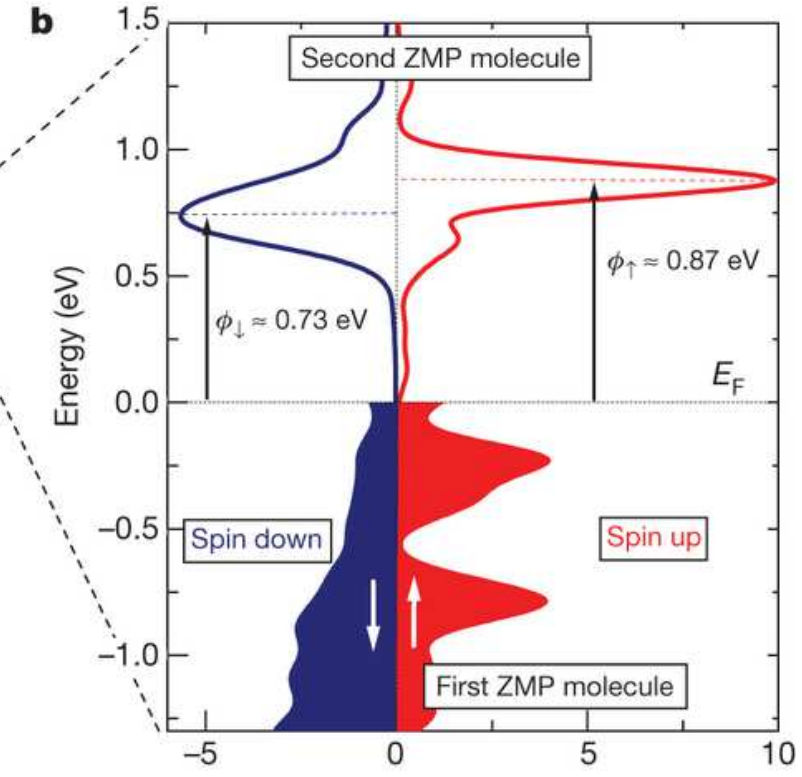

PDOS (states per eV)

\begin{tabular}{|c|c|}
\hline Axis & $\begin{array}{c}\text { MAE } \\
(\mathrm{meV})\end{array}$ \\
\hline$c$ & 0 \\
\hline$b$ & 1.715 \\
\hline$a$ & 2.54 \\
\hline$(a+b) / \sqrt{ } 2$ & 2.975 \\
\hline
\end{tabular}

a, Spin-resolved PDOS of the $p$-states for the first-layer (green) and second-layer (purple) ZMP molecule of the relaxed interface system. Interface $p_{z}-d$ hybridization creates spin-unbalanced electronic structure in the two molecules. $\mathbf{b}$, Zoomed-in view of the PDOS in a in the energy interval $[-1.5,1.5 \mathrm{eV}]$. The LUMO of the second ZMP molecule is spin-split by $0.14 \mathrm{eV}$, leading to a difference 
Karthik V. Raman, Alexander M. Kamerbeek, Arup Mukherjee, Nicolae Atodiresei, Tamal K. Sen, Predrag Lazić, Vasile Caciuc, Reent Michel, Dietmar Stalke, Swadhin K. Mandal, Stefan Blügel, Markus Münzenberg \& Jagadeesh S. Moodera (2013.), "Interface-engineered templates for molecular spin memory devices", Nature, Vol. 493, No. 7433, pp. 509-513. DOI: 10.1038/nature11719

in the barrier height for the two spin channels. c, Calculation of MAE due to surface magnetocrystalline anisotropy for the organometallic supramolecule comprising seven hybridized Co atoms (gold) with the ZMP molecular dimer as a model system. The MAE of the supramolecule is calculated (table) for three different in-plane magnetization orientations with respect to the out-of plane magnetization defined here as the $c$ axis.

Our calculations show that the distance between the phenalenyl rings in the magnetic molecule and the molecule above is $\sim 3.26 \AA$, which is smaller than the sum of the van der Waals radii of the carbon atoms ${ }^{11}$. This suggests a weak chemical bonding of the molecular $\pi$-dimer. The PDOS of the second-layer ZMP molecule resembles the molecular-type discrete energy levels above and below the Fermi level $\left(E_{F}\right)$. Although the calculations show that the second ZMP molecule has no magnetic moment, the $\pi-\pi$ interaction with the magnetic molecule creates a spin-unbalanced electronic structure. The first energy level above $E_{\mathrm{F}}$ (the lowest unoccupied molecular orbital (LUMO) level) is observed to be spin-split by $0.14 \mathrm{eV}$ (Fig. 4b), defining two different barrier heights for spin tunnelling injection: a lower one for spin-down electrons $\left(\varphi_{\downarrow}\right)$ of $0.73 \mathrm{eV}$ and a higher one for spin-up electrons $\left(\varphi_{\uparrow}\right)$ of $0.87 \mathrm{eV}$, leading to a spin-dependent interface resistance. It is well known that conventional spin-filter tunnelling of this type (using magnetic semiconductors such as EuS, EuO, and so on) can be highly efficient ${ }^{21}$.

The independent switching of the interface magnetic layer close to room temperature implies a significantly large surface magnetic anisotropy energy (MAE $\sim 10 k T$ ). Because the molecule is composed of low-atomic-number elements that show weak spin-orbit coupling, the magnetic molecule alone cannot account for such large MAE. DFT study shows that the induced magnetic moment in the adsorbed ZMP molecule depends directly on the magnetic interaction with the surface Co layer. Therefore, the observation of IMR response suggests interface hybridization as the source of MAE. We expect an enhancement in $M A E^{5,27}$ of the Co surface ( $K_{\text {sur, }}$ in units of per surface atom) and significant weakening of the magnetic exchange interaction between the surface Co layer and the layers below it $\left(J_{\perp}\right)$. Calculations of MAE were performed on a model organometallic supramolecule, comprising seven Co atoms with the molecular dimer on top. The calculated MAE for different in-plane magnetization orientations is listed in Fig. 4c, showing a large enhancement of in-plane $K_{\text {sur, }}$ $\sim 180 \mu \mathrm{eV}$ per surface Co atom as compared to bulk anisotropy energy of $19 \mu \mathrm{eV}$ per atom ${ }^{28}$. This enhancement in $K_{\text {sur }}$ is in line with our experimental observations. Further, the anisotropy tends to be uniaxial, with the easy axis along the field cooled orientation (see Supplementary Information).

Magnetic exchange interactions at the ferromagnet surface can be weaker than within the bulk. Owing to reduction in the moment and fewer magnetic neighbours at the interface, the interatomic magnetic exchange coupling $(J)$ can reduce considerably, and become comparable to or even lower than the surface anisotropy ${ }^{29,}{ }^{30}$ : that is, $J / K_{\text {sur }} \sim<1$. In such cases, the bulk magnetization can switch before the surface magnetization ${ }^{28}$. For bulk f.c.c. Co, $J$ is computed to be $\sim 8.54$ meV per atom, consistent with the values reported by others ${ }^{28}$ (see Supplementary Information). For a hybridized Co surface this value is reduced to 1.34 meV per Co atom, corresponding to a $70 \%$ reduction in $J_{\perp}$ compared to a clean Co surface (see Supplementary Information for $J_{\perp}$ calculation). The strong effect of interface hybridization on the properties of the Co surface layer is clearly evident from these studies, signifying a large increase in the ratio $K_{\text {sur }} / J_{\perp}$ compared to the bulk $(K / J \approx 0.002)^{28}$. This phenomenon explains the switching response of the interface magnetic layer responsible for 
the observed IMR. Deeper understanding of the interface mechanisms responsible for magnetic switching and the occurrence of IMR is a subject for future studies.

In order to use the adsorbed magnetic molecule as a source of information or a magnetic 'bit', one needs to be able to independently switch the moment of the adsorbed molecule. The use of phenalenyl-derived molecules provides a unique advantage in this regard. Using chemical synthesis, one could tune the chemical state (that is, cation, radical or anion) as well as the topological state of the phenalenyl moiety in the molecule, and control the charge transfer and hybridization effects with the ferromagnet surface. In the case of ZMP, the observation of an asymmetric magnetoresistance behaviour near room temperature (Fig. $3 b$ ) suggests a weakening of magnetic exchange coupling $J_{\text {Co-zMP, }}$ leading to independent magnetization switching of the magnetic molecular dimer from parallel to antiparallel alignment and vice-versa with respect to the hybridized surface Co layer. The above results demonstrate the potential for engineering the interface magnetic exchange interaction at the molecular level to control the independent hysteretic response of the adsorbed magnetic molecule. In addition, the IMR response provides a robust mechanism to probe and manipulate the spin state of the magnetic molecule, paving way to the development of room temperature molecular memory devices. Furthermore, the spin structure of the phenalenyl ligands can take the role of bus qubits ${ }^{9}$ in tailor-made molecular networks for realizing future molecular quantum information processing systems.

\section{Methods}

\section{Synthesis of ZMP}

(ZMP has CCDC structure no. 859902.) As a starting material 9-hydroxy-1-oxophenalene was synthesized and dissolved $(0.196 \mathrm{~g}, 1.0 \mathrm{mmol})$ in tetrahydrofuran $(20 \mathrm{ml})$ and $\mathrm{ZnMe}_{2}(1.2 \mathrm{M}$ in toluene; $1.0 \mathrm{ml}, 1.2 \mathrm{mmol}$ ) was added. The resulting solution was concentrated under reduced pressure and kept at $-20^{\circ} \mathrm{C}$ for a day to develop suitable crystals (see Supplementary Information for details).

\section{Device preparation}

The thin film devices were fabricated on pre-cleaned glass substrates in a high vacuum thermal deposition chamber $\left(10^{-7}\right.$ torr) with in situ shadow masking. The Co bottom electrode was grown on cooled substrates $(80 \mathrm{~K})$ using electron-beam evaporation. The ZMP organic layer and top electrodes were subsequently grown at room temperature. The junction area was $200 \mu \mathrm{m} \times 200 \mu \mathrm{m}$. The device was covered by a 10-nm-thick $\mathrm{Al}_{2} \mathrm{O}_{3}$ capping layer. In each pump-down 72 junctions were made, and about 15 such junction sets were investigated by varying the organic layer thickness and the interface as well as the type of top and bottom electrodes.

\section{DFT method}

Spin-polarized first-principles calculations were performed using density-functional theory (DFT) by employing the generalized gradient approximation (PBE, for Co atom $\mathrm{PBE}+\mathrm{U}$, with $U_{\text {eff }}=3.0 \mathrm{eV}$; see Supplementary Information for details) in a projector augmented plane- 
Karthik V. Raman, Alexander M. Kamerbeek, Arup Mukherjee, Nicolae Atodiresei, Tamal K. Sen, Predrag Lazić, Vasile Caciuc, Reent Michel, Dietmar Stalke, Swadhin K. Mandal, Stefan Blügel, Markus Münzenberg \& Jagadeesh S. Moodera (2013.), "Interface-engineered templates for molecular spin memory devices", Nature, Vol. 493, No. 7433, pp. 509-513. DOI: 10.1038/nature11719

wave formulation as implemented in VASP code. The structural relaxations included the van der Waals forces in a semi-empirical approach.

\section{References}

1. Verdaguer, M. Molecular electronics emerges from molecular magnetism. Science 272, 698699 (1996)

2. Miyamachi, T. et al. Robust spin crossover and memristance across a single molecule. Nature Commun. 3, 938 (2012)

3. Rocha, A. R. et al. Towards molecular spintronics. Nature Mater. 4, 335-339 (2005)

4. $\quad$ Leuenberger, M. N. \& Loss, D. Quantum computing in molecular magnets. Nature 410, 789793 (2001)

5. Mannini, M. et al. Magnetic memory of a single-molecule quantum magnet wired to a gold surface. Nature Mater. 8, 194-197 (2009)

6. Sessoli, R., Gatteschi, D., Caneschi, A. \& Novak, M. A. Magnetic bistability in a metal-ion cluster. Nature 365, 141-143 (1993)

7. Fuchs, G. D., Burkard, G., Klimov, P. V. \& Awschalom, D. D. A quantum memory intrinsic to single nitrogen-vacancy centres in diamond. Nature Phys. 7, 789-793 (2011)

8. Ardavan, A. et al. Will spin-relaxation times in molecular magnets permit quantum information processing? Phys. Rev. Lett. 98, 057201 (2007)

9. Morita, Y., Suzuki, S., Sato, K. \& Takui, T. Synthetic organic spin chemistry for structurally welldefined open-shell graphene fragments. Nature Chem. 3, 197-204 (2011)

10. Haddon, R. C. Design of organic metals and superconductors. Nature 256, 394-396 (1975)

11. Hicks, R. G. What's new in stable radical chemistry? Org. Biomol. Chem. 5, 1321-1338 (2007)

12. Itkis, M. E. et al. Magneto-opto-electronic bistability in a phenalenyl-based neutral radical. Science 296, 1443-1445 (2002)

13. Wende, H. et al. Substrate-induced magnetic ordering and switching of iron porphyrin molecules. Nature Mater. 6, 516-520 (2007)

14. Manriquez, J. M., Yee, G. T., Mclean, R. S., Epstein, A. J. \& Miller, J. S. A room-temperature molecular/organic-based magnet. Science 252, 1415-1417 (1991)

15. Herrmann, C., Solomon, G. C. \& Ratner, M. A. Organic radicals as spin filters. J. Am. Chem. Soc. 132, 3682-3684 (2010)

16. Gütlich, P. \& Goodwin, H. A. Spin Crossover in Transition Metal Compounds I (Springer, 2004)

17. Morita, Y. et al. Organic tailored batteries materials using stable open-shell molecules with degenerate frontier orbitals. Nature Mater. 10, 947-951 (2011)

18. Xiong, Z. H., Wu, D., Vardeny, Z. V. \& Shi, J. Giant magnetoresistance in organic spin-valves. Nature 427, 821-824 (2004)

19. Santos, T. S. et al. Room-temperature tunnel magnetoresistance and spin-polarized tunneling through an organic semiconductor barrier. Phys. Rev. Lett. 98, 016601 (2007)

20. Shim, J. H. et al. Large spin diffusion length in an amorphous organic semiconductor. Phys. Rev. Lett. 100, 226603 (2008)

21. Moodera, J. S., Santos, T. S. \& Nagahama, T. The phenomena of spin-filter tunneling. J. Phys. Condens. Matter 19, 165202 (2007)

22. Gould, C. et al. Tunneling anisotropic magnetoresistance: a spin-valve-like tunnel magnetoresistance using a single magnetic layer. Phys. Rev. Lett. 93, 117203 (2004)

23. Grünewald, M. et al. Tunneling anisotropic magnetoresistance in organic spin valves. Phys. Rev. B 84, 125208 (2011) 
24. Atodiresei, N. et al. Design of the local spin polarization at the organic-ferromagnetic interface. Phys. Rev. Lett. 105, 066601 (2010)

25. Raman, K. V. et al. Effect of molecular ordering on spin and charge injection in rubrene. Phys. Rev. B 80, 195212 (2009)

26. Sanvito, S. Molecular spintronics: The rise of spinterface science. Nature Phys. 6, 562-564 (2010)

27. Gambardella, P. et al. Supramolecular control of the magnetic anisotropy in two-dimensional high-spin Fe arrays at a metal interface. Nature Mater. 8, 189-193 (2009)

28. Dimitrov, D. A. \& Wysin, G. M. Magnetic properties of spherical fcc clusters with radial surface anisotropy. Phys. Rev. B 51, 11947-11950 (1995)

29. Siegmann, H. C. Surface and 2D magnetism. J. Phys. Condens. Matter 4, 8395-8434 (1992)

30. Miller, J. S. \& Drillon, M. Magnetism: Molecules to Materials Vol. 3, Magnetism: Nanosized Magnetic Materials (Wiley-VCH, 2002)

\section{Acknowledgements}

We thank J. M. D. Coey of Trinity College, Ireland, for discussions. K.V.R. and J.S.M. were supported by the Office of Naval Research (ONR grant N00014-09-1-0177) and the National Science Foundation (grants DMR 0504158 and ULFR 09-0532-01). A.M.K. thanks the University of Groningen for partial financial support during his stay at MIT. N.A. and V.C. thank the Julich Supercomputing Centre, Forschungszentrum Julich (Germany), for performing calculations on JUROPA and JUGENE supercomputers. A.M. and T.K.S. thank IISER-Kolkata and CSIR, India, respectively, for research fellowships. S.K.M. thanks CSIR (sanction no. 01(2369)/10/EMR-II), India, for financial support. M.M. thanks the German Science foundation for support within SFB 602 and SPP 1538, and S. Demeshko for SQUID measurements. D.S. and R.M. thank the Deutsche Forschungsgemeinschaft (DFG) Priority Programme 1178 and the Danish National Research Foundation (DNRF) funded Center for Materials Crystallography (CMC) for support, and the Land Niedersachsen for providing a fellowship in the Catalysis for Sustainable Synthesis (CaSuS) Ph.D. program. J.S.M., M.M, S.K.M. and D.S. thank the Göttingen-Kolkata 'Open shell systems (G-KOSS)' initiative for supporting the collaboration.

\section{Author information}

These authors contributed equally to this work: Karthik V. Raman \& Alexander M. Kamerbeek

\section{Affilations}

1. Francis Bitter Magnet Laboratory, Massachusetts Institute of Technology, Massachusetts 02139, USA

Karthik V. Raman, Alexander M. Kamerbeek \& Jagadeesh S. Moodera

2. Department of Materials Science and Engineering, Massachusetts Institute of Technology, Massachusetts 02139, USA

Karthik V. Raman 
3. Department of Chemical Sciences, Indian Institute of Science Education and Research (IISER)-Kolkata, Mohanpur 741252, India

Arup Mukherjee, Tamal K. Sen \& Swadhin K. Mandal

4. Peter Grünberg Institut and Institute for Advanced Simulation, Forschungszentrum Jülich and JARA, 52425 Jülich, Germany

Nicolae Atodiresei, Vasile Caciuc \& Stefan Blügel

5. Theoretical Physics Division, Rudjer Boskovic Institute, PO Box 180, HR-10002 Zagreb,

Croatia

Predrag Lazić

6. Institut für Anorganische Chemie, Universität Göttingen, Göttingen 37077, Germany

Reent Michel \& Dietmar Stalke

7. Physikalisches Institut, Georg-August-Universität Göttingen, Göttingen 37077, Germany Markus Münzenberg

8. Physics Department, Massachusetts Institute of Technology, Massachusetts 02139, USA Jagadeesh S. Moodera

9. Present address: IBM India Research Laboratory, Bangalore 560045, India (K.V.R.); Institute for Advanced Materials, University of Groningen, Nijenborgh 4, 9747 AG, Groningen, The Netherlands (A.M.K.).

Karthik V. Raman \& Alexander M. Kamerbeek

\section{Contributions}

S.K.M., M.M. and J.S.M. designed the original research approach; A.M. and S.K.M. developed molecule synthesis; A.M.K., A.M., T.K.S., S.K.M., R.M. and D.S. performed material characterization; K.V.R. and A.M.K. prepared devices, performed transport experiments and analysed transport and magnetic characterization; N.A., P.L. and V.C. performed DFT calculations; K.V.R., A.M.K., N.A. and J.S.M. developed the organic spin-filter model; K.V.R., N.A. and J.S.M. developed the interface magnetic anisotropy model; K.V.R. coordinated collaboration; K.V.R., J.S.M. and M.M. wrote the manuscript; N.A., A.M.K., A.M. and S.K.M. provided the discussion and contributed to manuscript preparation. All authors discussed the experiments and commented on the manuscript.

\section{Coresponding authors}

Correspondence to: Karthik V. Raman (vkarthik@mit.edu) or Jagadeesh S. Moodera (moodera@mit.edu) or Nicolae Atodiresei (n.atodiresei@fz-juelich.de) or Swadhin K. Mandal (swadhin.mandal@iiserkol.ac.in). 